\title{
Evaluation of an Assessment System for the JRS 2005: A-DROP for the Management of CAP in Adults
}

\author{
Shigeru Kohno ${ }^{1}$, Masafumi Seki ${ }^{1}$, Akira Watanabe ${ }^{2}$ and the CAP Study Group
}

\begin{abstract}
Objective The Japanese Respiratory Society (JRS) last revised the guidelines for community-acquired pneumonia (CAP) in adults in 2005. These guidelines proposed new criteria (A-DROP) to assess the severity of pneumonia and to differentiate between typical bacterial pneumonia and atypical pneumonia. The goal of the present study was to evaluate the utility of the A-DROP criteria for these described purposes.

Methods An observational survey was conducted between July 2006 and March 2007, and patients with CAP were prospectively surveyed using consecutive enrollment methods.

Patients In total, 1,875 patients from 200 medical facilities throughout Japan were analyzed.

Results The JRS 2005 A-DROP system was a good indicator of mortality in the patient population, and these results were significantly correlated with the Pneumonia Severity Index (PSI) of the Infectious Disease Society of America (IDSA). Among the various factors characterized, ' $\mathrm{SpO}_{2}$ of $90 \%$ or less $\left(\mathrm{PaO}_{2}\right.$ of 60 Torr or less)' was the strongest predictor of mortality. In terms of the differential diagnosis between typical bacterial and atypical pneumonia, five of six JRS 2005 items were strongly and significantly correlated with a diagnosis of atypical pneumonia.
\end{abstract}

Conclusion The JRS 2005 A-DROP system was accurate and clinically useful for the assessment of the severity of pneumonia and for the differentiation between typical bacterial pneumonia and atypical pneumonia.

Key words: A-DROP system, bacterial pneumonia, atypical pneumonia, The Japanese Respiratory Society, guidelines, community-acquired pneumonia

(Intern Med 50: 1183-1191, 2011)

(DOI: 10.2169/internalmedicine.50.4651)

\section{Introduction}

Community-acquired pneumonia (CAP) is a common and clinically important infectious disease that affects adults worldwide. The clinical symptoms and consequences of CAP may vary with patient age, severity of the underlying disease, and causal microorganisms, which can sometimes confound clinical assessment, patient triage, and determination of the prognosis at initial presentation (1-4).

Medical care guidelines for CAP were sequentially published and revised in Europe and the USA in the 1990s (3-5). The Japanese Respiratory Society (JRS) also released their "Basic Concepts in the Medical Care of CAP in Adults" (JRS 2000) (6), with subsequent revision under the title, "Guidelines for the Management of CAP in Adults" (JRS 2005) (7). These guidelines were designed to apply to the general population and are now regularly used by physicians specializing in medical fields other than pulmonology.

The JRS proposed relatively simple criteria to assess the severity of CAP. This strategy, known as the "A-DROP system" (7), was a modified version of the "CURB-65" system of the British Thoracic Society (BTS) and was intended to help predict patient mortality and to help select the appropriate venue for ongoing care; i.e., outpatient treatment or consideration of hospitalization for mild to moderate cases versus admission to the intensive care unit for moderate to severe cases $(7,8)$. Indeed, several publications showed that CURB-65 was handier than IDSA/PSI and provided suffi-

${ }^{1}$ Department of Molecular Microbiology and Immunology (2nd Internal Medicine), Nagasaki University Graduate School of Biomedical Sciences, Nagasaki University Hospital, Japan and ${ }^{2}$ Institute Research Division for Development of Anti-Infective Agents, Tohoku University, Japan Received for publication October 8, 2010; Accepted for publication February 8, 2011

Correspondence to Dr. Shigeru Kohno, s-kohno@nagasaki-u.ac.jp 
cient data for clinical use (9).

The JRS also regards the differential diagnosis of typical bacterial pneumonia versus atypical pneumonia to be important, especially because macrolide-resistant pneumococcus is highly prevalent in the Japan, making macrolides a poor first-line therapy for bacterial pneumonia $(6,7,10-12)$ and because respiratory fluoroquinolones are not given via the intravenous route in Japan $(7,8)$. Therefore, the JRS has proposed diagnostic criteria to differentiate between typical bacterial and atypical pneumonia and has recommended penicillins or macrolides as first-line therapy for typical bacterial pneumonia or atypical pneumonia, respectively (7).

Despite the widespread use of these guidelines, there has been no definitive examination of the correlation between JRS A-DROP assessed severity of pneumonia and patient outcomes or of the utility of JRS differentiation of typical bacterial pneumonia versus atypical pneumonia. Therefore, the goal of the present study was to show some conclusive or confirming result to evaluate the accuracy and clinical utility of the JRS A-DROP system to assess the severity of pneumonia and the differential diagnosis between typical bacterial pneumonia and atypical pneumonia.

\section{Materials and Methods}

\section{Subjects}

Subjects were adult patients with CAP treated by physicians specializing in respiratory diseases among 200 medical facilities throughout Japan between July 2006 and March 2007. A prospective observation approach was used in this cohort study.

Patients were older than 16 years and had clinical symptoms of cough, sputum production, and fever. In all cases, chest X-ray examination or computed tomograph (CT) scans of the chest revealed shadows corresponding with acute infiltrates. Patients who had developed pneumonia more than 48 hours after admission (hospital-acquired pneumonia) and those who showed signs of improvement due to previous antimicrobial treatment were not included. This trial was approved by the institutional review board of each participating medical facility, including Nagasaki University and Tohoku University, and all patients were given an explanation of this observational study in advance and provided full written informed consent to participate in this study.

A continuous enrollment system was adopted to minimize treating physician bias. All patients meeting the inclusion criteria after initiation of the survey were enrolled in this study until a predetermined number was reached at each medical facility.

\section{Assessment of severity according to JRS 2005, and stratification of risk according to Infectious Disease of America (IDSA) guidelines}

The assessment of severity by the JRS 2005 A-DROP system is based on five clinical features: age (A), dehydra- tion (D), respiration (R), orientation $(\mathrm{O})$ and blood pressure (P). In this study, cases were regarded as "mild" with none of the five criteria met, as "moderate" with one or two of the criteria met, as "severe" with three of the criteria met, and as "extremely severe" with four or five of the criteria met (7). Any patient showing signs of shock or altered mental status was deemed to have extremely severe pneumonia, regardless of the number of criteria fulfilled.

Risk stratification was performed according to IDSA guidelines by calculating the Pneumonia Severity Index (PSI) as follows (4): a score of zero = low-risk class I; a score $\leq 70$ points $=$ low-risk class II; a score between 71 and 90 points = low-risk class III; a score between 91 and 130 points $=$ intermediate-risk; a scores $>130$ points $=$ highrisk. In some cases, particular observations and examinations were not conducted, such as scores for these items were counted as zero for the calculations.

In order to compare the degree of severity as categorized by JRS 2005 A-DROP with the risk classes specified by IDSA, the "mild" designation was paired with the low-risk classes I-III, the "moderate" designation was paired with the intermediate-risk class, and the "severe" and "extremely severe" designations were paired with the high-risk class. For scoring, mild, moderate, severe and extremely severe corresponded to one, two, three and four points, respectively.

Outcomes were evaluated 30 days after initiation of the first-line therapy, and the survival rate was estimated.

\section{Microbiological data analysis}

Sputum samples were obtained for bacterial testing prior to initiating first-line therapy. Causative organisms were identified by taking into account the number of viable cells and known pathogenicity $(7,8)$. Moreover, the pathogens of atypical pneumonia were identified by acute-phase serologic test (complement fixation and particle agglutination methods), 'IMMUNOCARD test' (serum enzyme immunoassay methods) (Nihon TFB Co., Ltd., Tokyo, Japan).

\section{Differential diagnosis between typical bacterial pneumonia and atypical pneumonia}

Criteria used to assess for the presence of atypical pneumonia in patients with unknown causal microorganisms included: under 60 years of age, no or minor underlying disease, stubborn cough, poor chest auscultatory findings, no sputum or no identified aetiological agent by rapid diagnosis, and a peripheral white blood cell count below 10,000/ $\mu \mathrm{L}$ (7). The patients were classified as having either "suspected atypical pneumonia", when four or more of the six criteria were met, or having "suspected typical bacterial pneumonia" when less than four of the criteria were met.

An earlier version of the JRS guidelines (JRS 2000) also included three other factors (cluster of pneumonia among family members or close associates, absence of tachycardia in the context of fever, and ground glass opacity or skip lesion on chest X-ray), which were also assessed in the present study (6). 


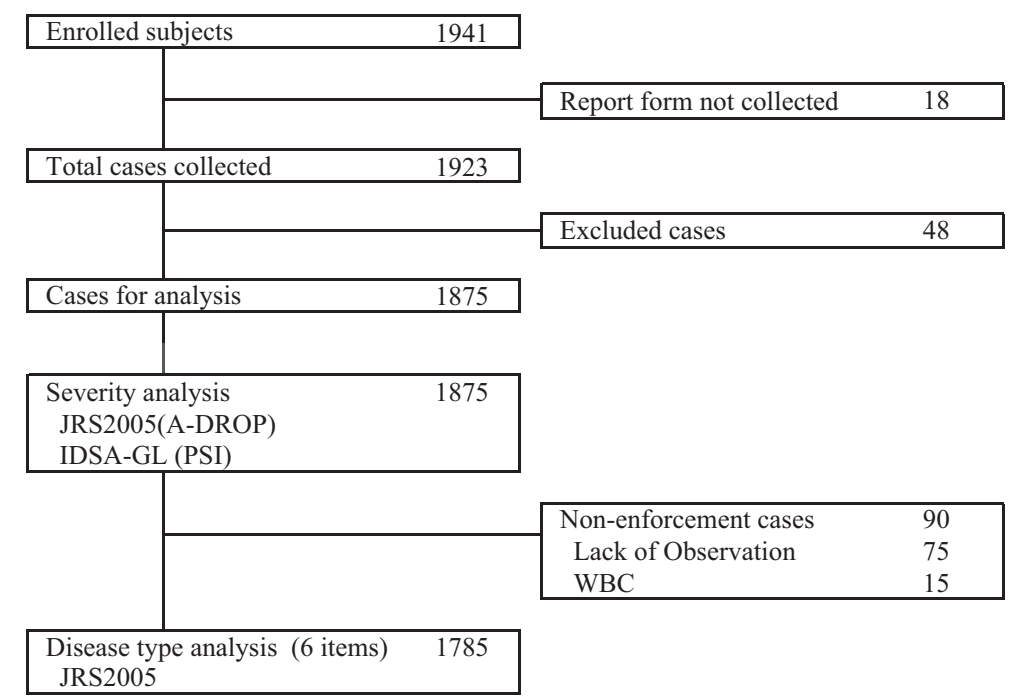

Figure 1. Case breakdown.

Table 1. Patient Characteristics according to Severity Classification

\begin{tabular}{|c|c|c|c|c|c|c|}
\hline \multirow{2}{*}{ Indicators (A-DROP) } & No & \multirow{2}{*}{ Yes } & \multicolumn{4}{|c|}{ Severity classification } \\
\hline & Total $(\%)$ & & Mild & Moderate & Severe & Extremely severe \\
\hline Male aged 70 years or older female aged 75 years or older & $1080(57.6)$ & 795 (42.4) & 0 & 598 & 154 & 43 \\
\hline BUN of $21 \mathrm{mg} / \mathrm{dL}$ or more, or presence of dehydration & $1448(77.2)$ & $427(22.8)$ & 0 & 236 & 148 & 43 \\
\hline $\mathrm{SpO}_{2}$ of $90 \%$ or less $\left(\mathrm{PaO}_{2}\right.$ of 60 Torr or less $)$ & $1441(76.9)$ & $434(23.1)$ & 0 & 245 & 146 & 43 \\
\hline Disturbance of consciousness & $1780(94.9)$ & $95(5.1)$ & 0 & 22 & 35 & 38 \\
\hline Blood pressure (systolic) of $90 \mathrm{mmHg}$ or less & $1836(97.9)$ & $39(2.1)$ & 0 & 13 & 9 & 17 \\
\hline Total $(\%)$ & & 1875 & $857(45.7)$ & $808(43.1)$ & $164(8.7)$ & $46(2.5)$ \\
\hline
\end{tabular}

\section{Statistical analysis}

Relative coefficients were used for all statistical analyses, which were conducted using the SAS software program (SAS, version 9.1; SAS Institute Inc., Cary, NC). Comparisons between severity and the mortality risk were assessed using Receiver Operatorating Characteristic (ROC) analysis. Relationships between items were assessed using multiple logistic regression analysis. $\mathrm{p}<0.05$ was considered statistically significant.

\section{Results}

\section{Patient population}

In total, 1,941 patients treated among 200 medical facilities throughout Japan were enrolled in this survey, and 1,923 responses were collected. The respondents included two patients who were younger than 16 years, 14 who did not undergo a chest $\mathrm{X}$-ray examination or had no infiltrates on chest X-ray films at the time of initial consultation, and 32 with diseases other than the target infection. Therefore, 1,875 patients were analyzed for severity (Fig. 1). In addition, 90 cases were excluded from the differential diagnosis analysis (i.e. typical bacterial pneumonia versus atypical pneumonia) due to lack of appropriate data.

Use of the JRS 2005 A-DROP system to assess disease severity resulted in designation of $857(45.7 \%)$ cases as mild, $808(43.1 \%)$ cases as moderate, $164(8.7 \%)$ cases as severe, and $46(2.5 \%)$ cases as extremely severe (Table 1$)$. Mortalities of these groups were $0 \%, 3.1 \%, 9.9 \%$ and $19.6 \%$, respectively (data not shown).

In the assessment system for the differential diagnosis between typical bacterial pneumonia and atypical pneumonia, $1,213(68.3 \%)$ were suspected to have typical bacterial pneumonia, and $562(31.7 \%)$ cases were suspected to have atypical pneumonia (Table 2).

\section{Comparison of pneumonia severity classifications by JRS 2005 A-DROP and IDSA PSI}

Using the PSI, 1,338 (71.4\%), 425 (22.7\%) and 112 (6.0\%) of the total 1,875 patients were assessed as having mild, moderate and severe pneumonia, respectively (Table 3). Mortalities of these groups were $0.4 \%, 5.9 \%$ and $18.0 \%$, respectively (data not shown).

Nearly all cases of pneumonia assessed as mild by the ADROP were also assessed as mild by the PSI (99.6\%). Among the 808 cases of pneumonia assessed as moderate by the A-DROP, $482(59.6 \%)$ were assessed as mild, 310 $(38.4 \%)$ were assessed as moderate, and $16(2.0 \%)$ were assessed as severe by the PSI. Among the total 164 cases of pneumonia assessed as severe by the A-DROP, one $(0.6 \%)$ was assessed as mild, $105(64.0 \%)$ were assessed as moderate, and $58(35.4 \%)$ were assessed as severe by the PSI. 
Table 2. Characteristics of Patients according to Suspected Type of Pneumonia

\begin{tabular}{lrrr}
\hline \multicolumn{1}{c}{ Items used to differentiate } & Atypical pneumonia & Bacteria pneumonia & Total \\
& suspected & suspected & $667(100 \%)$ \\
\hline Under 60 years of age & $440(66.0 \%)$ & $227(34.0 \%)$ & $1347(100 \%)$ \\
No or minor underlying disease & $549(40.8 \%)$ & $798(59.2 \%)$ & $548(100 \%)$ \\
Stubborn cough & $377(68.8 \%)$ & $171(31.2 \%)$ & $743(100 \%)$ \\
Poor chest auscultatory findings & $462(62.2 \%)$ & $281(37.8 \%)$ & $762(100 \%)$ \\
No sputum or no identified aetiological agent by rapid diagnosis & $422(55.4 \%)$ & $340(44.6 \%)$ & $851(100 \%)$ \\
A peripheral white blood cell count below $10,000 / \mu \mathrm{L}$ & $452(53.1 \%)$ & $399(46.9 \%)$ & $28(100 \%)$ \\
Cluster of pneumonia among family members or close associates & $19(67.9 \%)$ & $9(32.1 \%)$ & $23(100 \%)$ \\
Absence of tachycardia in the context of fever & $10(43.5 \%)$ & $13(56.5 \%)$ & $214(100 \%)$ \\
Ground glass opacity or skip lesion on chest X-ray & $124(57.9 \%)$ & $90(42.1 \%)$ & $1775(100 \%)$ \\
Total & $562(31.7 \%)$ & $1213(68.3 \%)$ & $17 \%$ \\
\hline
\end{tabular}

*10 cases with positive Legionella urinary antigen testing were excluded

Table 3. Comparison of Pneumonia Severity Classification by JRS 2005 (A-DROP) and IDSA (PSI)

\begin{tabular}{|c|c|c|c|c|c|c|c|c|}
\hline & \multirow{2}{*}{\multicolumn{2}{|c|}{ Severity }} & \multicolumn{6}{|c|}{ JRS2005 (A-DROP) } \\
\hline & & & \multicolumn{2}{|c|}{ Mild } & Moderate & Severe & \multirow{2}{*}{$\begin{aligned} & \text { Extremely } \text { severe } \\
& 1(2.2 \%)\end{aligned}$} & \multirow{2}{*}{$\begin{array}{c}\text { Total }(\%) \\
1338(71.4 \%)\end{array}$} \\
\hline & Mild & I-III & 854 & $(99.6 \%)$ & $482(59.6 \%)$ & $1 \quad(0.6 \%)$ & & \\
\hline IDSA & Moderate & IV & 3 & $(0.4 \%)$ & $310(38.4 \%)$ & $105(64.0 \%)$ & $7(15.2 \%)$ & $425(22.7 \%)$ \\
\hline (PSI) & Tota & $(\%)$ & 857 & $(100 \%)$ & $808 \quad(100 \%)$ & $164(100 \%)$ & $46(100 \%)$ & $1875(100 \%)$ \\
\hline
\end{tabular}

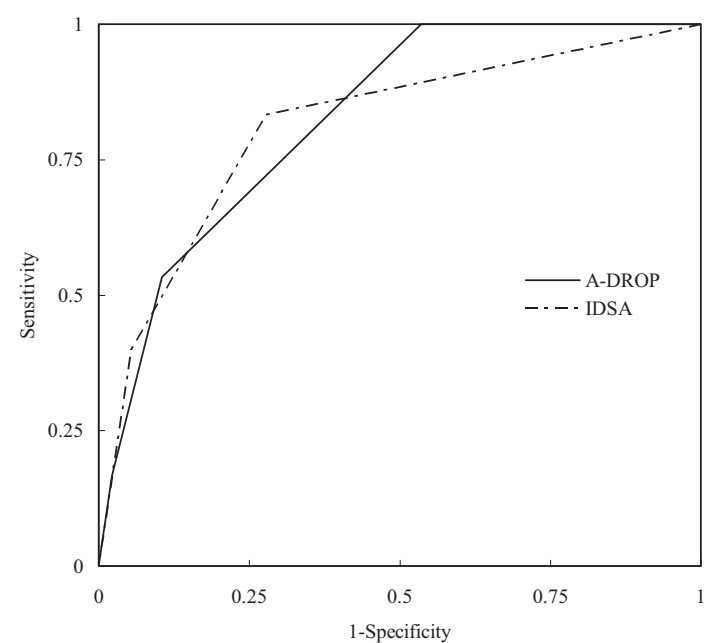

Figure 2. The ROC curve for 30-days mortality for ADROP and PSI. The area under the ROC curves was 0.824 [95\% confidence intervals $(\mathrm{CI}): \mathbf{0 . 8 2 2 - 0 . 8 2 7}, \mathrm{p}<0.001]$ for $\mathrm{A}$ DROP and 0.811 [95\% confidence intervals (CI): 0.807-0.814, $\mathbf{p}<0.001]$ for PSI.

Among the 46 cases of pneumonia assessed as extremely severe by the A-DROP, 38 (82.6\%) were assessed as severe by the PSI. Assessment of disease severity by A-DROP correlated significantly with that by PSI ( $\mathrm{r}=0.6781, \mathrm{p}<0.0001$ : Fig. 2).

\section{Relationship between mortality and JRS 2005 A- DROP pneumonia severity indicators}

The relationship between the five A-DROP indicators and mortality was characterized. Logistic regression ' $\mathrm{SpO}_{2}$ of $90 \%$ or less $\left(\mathrm{PaO}_{2}\right.$ of 60 Torr or less)' as the strongest predictor of mortality [adjusted odds ratio (OR), 7.034; $95 \%$ confidence interval (CI), 2.689-18.400; p<0.0001, Fig. 3]. In fact, this indicator was associated with $5.6 \%$ (24 cases) of all deaths.

The other four indicators also tended to be associated with mortality, with 'Disturbance of consciousness' accounting for the greatest proportion there of $(8.4 \%)$, and 'male aged 70 years or older, female aged 75 years or older' for the least $(3.2 \%)$.

\section{JRS 2005 A-DROP system and differential diagnosis between typical bacterial pneumonia and atypical pneumonia}

Finally, correlations between JRS 2005 A-DROP system and a diagnosis of typical bacterial pneumonia or atypical pneumonia were characterized by logistic regression. The incidence of pathogens was as follows; $S$. pneumoniae, $36.5 \%$ (323 isolates); M. pneumoniae, $24.2 \%$ (214 isolates); $C$. pneumoniae, $11.9 \%$ (105 isolates); $H$. influenzae, $11.6 \%$ (103 isolates); M. catarrhalis, 3.7\% (33 isolates), and Legionella spp., $1.1 \%$ (10 isolates) (Table 4). The atypical pneumonia of pathogens was as follows; Mycoplasma inspection positivity 214 patients; Chlamydia examination positivity 105 patients (Table 4). The bacteria unidentified cases was $58.1 \%$ (1,089 patiens). Six of nine items were significantly correlated with the differential diagnosis between atypical and bacterial pneumonia (Fig. 4). However, 'cluster of pneumonia among family members or close associates' and 'absence of tachycardia in the context of fever' were not significantly related to the differential diagnosis ( $\mathrm{p}=0.2967$ and 0.4115 , respectively).

\section{Discussion}

The JRS revised criteria assessed severity of pneumonia, published as the JRS 2005, are routinely used by general 


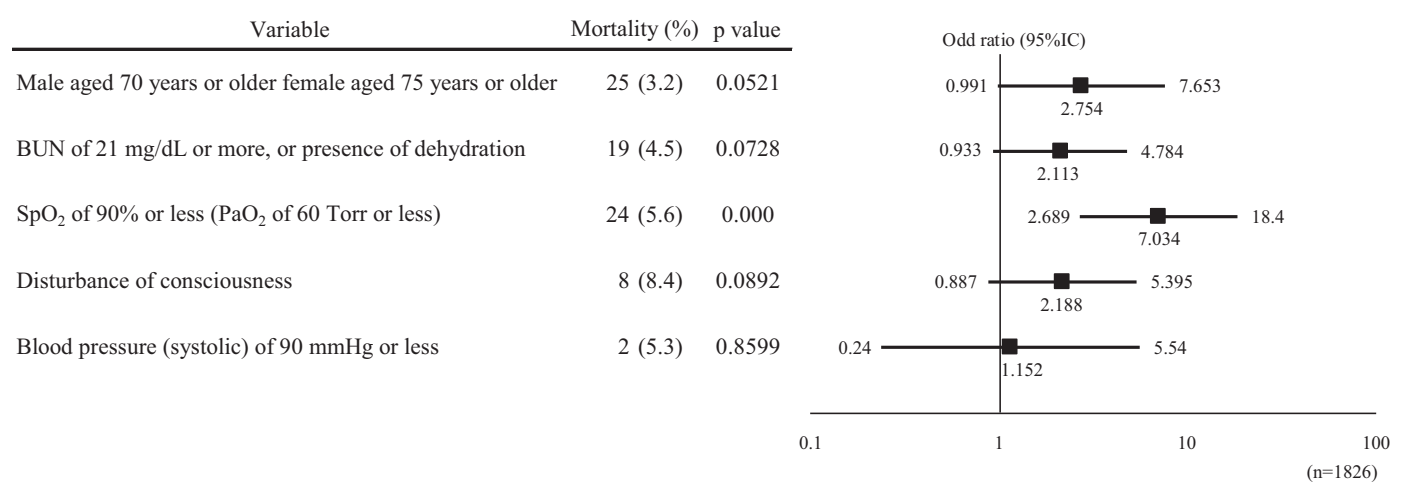

Figure 3. Multiple logistic regression analysis of the relationship between mortality and factors predicting severity of pneumonia severity according to JRS2005 (A-DROP). The 1,826 patients, which 49 cases with unknown outcome were excluded from the 1,875 patients, were analyzed.

Table 4. Details of Causative Organisms

\begin{tabular}{|c|c|c|}
\hline \multicolumn{2}{|c|}{ Causative organism } & Isolates \\
\hline \multirow[t]{5}{*}{ Gram positive } & Staphylococcus aureus & $22(2.5 \%)$ \\
\hline & Streptococcus pneumoniae & $323(36.5 \%)$ \\
\hline & Streptococcus anginosus group & $1(0.1 \%)$ \\
\hline & Streptococcus spp. & $12(1.4 \%)$ \\
\hline & Corynebacterium spp. & $2(0.2 \%)$ \\
\hline \multirow[t]{9}{*}{ Gram negative } & Moraxella catarrhalis & $33(3.7 \%)$ \\
\hline & Escherichia coli & $5(0.6 \%)$ \\
\hline & Klebsiella spp. & $25(2.8 \%)$ \\
\hline & Enterobacter aerogenes & $2(0.2 \%)$ \\
\hline & Morganella morganii & $1(0.1 \%)$ \\
\hline & Haemophilus influenzae & $103(11.6 \%)$ \\
\hline & Pseudomonas aeruginosa & $25(2.8 \%)$ \\
\hline & Acinetobacter baumannii & $1(0.1 \%)$ \\
\hline & Legionella spp. & $10(1.1 \%)$ \\
\hline \multicolumn{2}{|l|}{ Anaerobic bacterium } & $1(0.1 \%)$ \\
\hline \multirow[t]{3}{*}{ Atypical bacterium } & Chlamydia pneumoniae & $105(11.9 \%)$ \\
\hline & Mycoplasma pneumoniae & $214(24.2 \%)$ \\
\hline & Total & $885(100.0 \%)$ \\
\hline
\end{tabular}

clinicians, and the scores obtained by these simple calculations appear to accurately reflect prognosis. Rather than using the complicated calculations associated with the IDSA PSI, the JRS composed their A-DROP criteria through modification of the simple BTS CURB-65 system.

In this study, $45.7 \%$ of CAP patients were designated as mild cases, $43.1 \%$ were designated as moderate cases, $8.7 \%$ were designated as severe cases, and $2.5 \%$ were designated as extremely severe cases. By contrast, Usui et al reported that $22.8 \%, 53.5 \%, 17.2 \%$, and $6.5 \%$ of their 523 CAP patients were categorized as mild, moderate, severe, and extremely severe cases, respectively (13). In addition, Tashiro et al reported that 25\%,48\%, 18\%, and 9\% of their 293 CAP patients were categorized as mild, moderate, severe, and extremely severe cases, respectively (14). The data from these two studies, which were conducted among local populations, were consistent with data from the present study, which drew patients from a nationwide population, and were thought to be representative of the Japanese population.

In confirming the validity of JRS 2005, we found a significant correlation between the severity assessment according to the A-DROP and the PSI, the reliability of which had already been established $(4,15)$. Mortality also showed a good correlation with assessment by the A-DROP, and the correlation coefficient was higher with the A-DROP than with the PSI. These results suggested that the well-selected characteristics employed in the A-DROP constitute an excellent system to assess prognosis.

Gomi et al reported that the classification of severity by A-DROP was correlated with that assessed by PSI and that the results of the two systems were comparable (16). However, while the PSI requires laboratory data to arrive at a score, the A-DROP requires only clinical criteria and is thus more practical for use and immediate application by clinicians.

The BTS CURB-65 system is similar to the A-DROP and is widely used. Several groups of investigators reported that this system is comparatively simple and it could identify severely ill individuals $(1,15,17)$. By contrast, the $\mathrm{pH}$ value and blood glucose levels needed to calculate the PSI were not measured in $74.0 \%$ and $31.0 \%$ of the cases in the present study, respectively (data not shown), indicating that this index may not be practical for routine clinical use. This notion was supported by Usui et al and Aujesky et al, who 


\begin{tabular}{lc}
\multicolumn{1}{c}{ Items used to differentiate } & $\mathrm{p}$ value \\
\hline Under 60 years of age & 0.000 \\
No or minor underlying disease & 0.0677 \\
Stubborn cought & 0.000 \\
Poor chest auscultatory findings & 0.0109 \\
No sputum or no identified aetiological agent by rapid diagnosis & 0.0111 \\
A peripheral white blood cell count below $10,000 / \mu \mathrm{L}$ & 0.000 \\
Cluster of pneumonia among family members or close associates & 0.2967 \\
Absence of tachycardia in the context of fever & 0.4115 \\
Ground glass opacity or skip lesion on chest X-ray & 0.0118
\end{tabular}

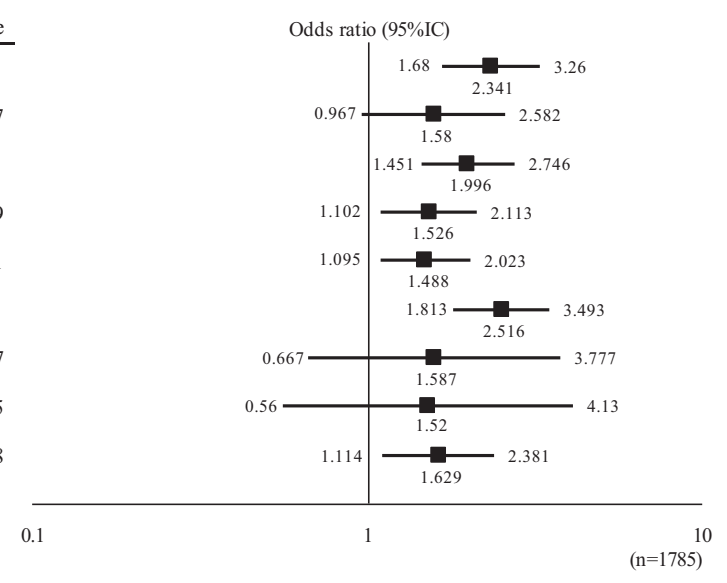

Figure 4. Multiple logistic regression analysis of the relationship between specific indicators and a suspected diagnosis of typical bacterial pneumonia versus atypical pneumonia.

suggested that the PSI was not well suited for typical daily clinical practice $(13,18)$. Although the use of pocket cards, personal electronic devices, or Internet support could facilitate the use of the PSI, data from the present study suggested that the A-DROP was more practical than the PSI.

Respiratory status, including such criteria as low $\mathrm{PaO}_{2}$, low $\mathrm{P} / \mathrm{F}$ ratio, high respiratory rate, and the need for mechanical ventilation, were included among several major severity scores, such as the PSI (4), CURB-65 (1), SMARTCOP (19), IDSA/ATS severity criteria 2007 (2), Espana et al criteria (20), SOAR criteria (21), ICU admission criteria in IDSA/ATS 2007 (2), and the I-ROAD scoring system for hospital-acquired pneumonia in JRS 2008 (8, 22). Data supporting the utility of respiratory status within those severity scores $(12,23)$, therefore, might also support the accuracy and utility of the A-DROP, which also incorporated respiratory status. Indeed, among the five indicators used in the ADROP, 'male aged 70 years or older, female aged 75 years or older' was most frequent, but ' $\mathrm{SpO}_{2}$ of $90 \%$ or less $\left(\mathrm{PaO}_{2}\right.$ of 60 Torr or less)' was the strongest predictor of mortality.

The present study also demonstrated a correlation between severity scores and sites-of-care. For example, treatment as an outpatient occurred in $48.1 \%$ of patients with mild pneumonia, $15.7 \%$ of patients with moderate pneumonia, $0.6 \%$ of patients with severe pneumonia, and $0 \%$ of patients with extremely severe pneumonia as classified by the A-DROP system (data not shown). Similarly, treatment as an inpatient occurred in $51.9 \%$ of patients with mild pneumonia, $84.2 \%$ of patients with moderate pneumonia, $99.4 \%$ of patients with severe pneumonia, and in $100 \%$ of patients with extremely severe pneumonia, as classified by the ADROP system (data not shown). We confirmed disease severity in A-DROP, but as a result many mild cases treated as outpatients in JRS 2005 were admitted for hospitalization. Because the Japanese insurance regime was more substantial than those of Western countries, it was speculated that most of the Japanese patients selected were not reluctant to be treated by hospitalization $(24,25)$. These data suggested that the A-DROP system provides a useful strategy to triage pa- tients to appropriate venues for ongoing care.

The correlation between mortality and chest X-ray findings and C-reactive protein (CRP) levels was also characterized but there were no significant correlations between these parameters (mortality versus chest $\mathrm{X}$-ray findings, $\mathrm{R}=0.184$; mortality versus $\mathrm{CRP}$ value, $\mathrm{R}=0.06$ ). However, we categorized $\mathrm{CRP} /$ chest X-ray infiltrations and analyzed the differences in prognosis in each category by Fisher's test. As a result, we found a significantly poorer prognosis in CRP $\geq$ $15 \mathrm{mg} / \mathrm{dL}$ and chest X-ray infiltrations $\geq 2 / 3$ (Table 5, 6). These results suggested that the prognoses were not clearly related to either CRP or chest X-ray infiltrations, but might be significantly worse in the category of high CRP and marked chest X-ray infiltrations. Therefore, we confirmed the relationship between mortality and 'five new items' that replaced 'Disturbance of consciousness' and 'Blood pressure' of A-DROP (Fig. 3) with CRP $\geq 15 \mathrm{mg} / \mathrm{dL}$ and chest $X$-ray infiltrations $\geq 2 / 3$. As a result, we found that the area under the ROC curve for five new items was 0.842 , which was also very good, however, not much higher than A-DROP (0.824) (Fig. 2).

This study represented the first assessment of the accuracy and validity of the JRS 2005 A-DROP criteria for the differential diagnosis between typical bacterial pneumonia and atypical pneumonia in the Japanese population. This protocol designated 562 cases $(31.7 \%)$ of suspected atypical pneumonia and 1,213 cases $(68.3 \%)$ of suspected bacterial pneumonia. Among the nine items assessed in this study (under 60 years of age; no or minor underlying disease; stubborn cough; poor chest auscultatory findings; no sputum or no identified aetiological agent by rapid diagnosis; a peripheral white blood cell count below 10,000/ $\mathrm{L}$; cluster of pneumonia among family members or close associates; absence of tachycardia in the context of fever, and ground glass opacity or skip lesion on chest X-ray), six were significant predictors of differentiating between typical bacterial pneumonia and atypical pneumonia, and five of these six items were employed for differential diagnosis in JRS 2005. These data strongly supported the utility of the JRS 
Table 5. Fisher's Test of the Relationship between CRP and Mortality

\begin{tabular}{clll}
\hline $\mathrm{CRP}(\mathrm{mg} / \mathrm{dL})$ & \multicolumn{1}{c}{ Mortality } & Fisher's test \\
\hline$<5$ & $0.4 \%(2 / 485)$ & & \\
$\geq 5,<10$ & $1.6 \%(8 / 493)$ & $1.1 \%(14 / 1307)$ & \\
$\geq 10,<15$ & $1.2 \%(4 / 329)$ & & \\
$\geq 15,<20$ & $2.8 \%(6 / 218)$ & & \\
$\geq 20,<25$ & $3.5 \%(4 / 113)$ & $2.9 \%(15 / 509)$ & \\
$\geq 25$ & $2.8 \%(5 / 178)$ & & \\
\hline unknown & $1.7 \%(1 / 59)$ & & \\
\hline
\end{tabular}

Table 6. Fisher's Test of the Relationship between $X$ ray Infiltrations and Mortality

\begin{tabular}{clll}
\hline $\mathrm{X}$ ray infiltrations & \multicolumn{2}{c}{ Mortality } & Fisher's test \\
\hline$<1 / 3$ & $0.8 \%(10 / 1219)$ & $0.9 \%(16 / 1719)$ & \\
$\geq 1 / 3,<2 / 3$ & $1.2 \%(6 / 500)$ & $\mathrm{p}=0.000$ \\
\hline $22 / 3$ & $9.7 \%(13 / 134)$ & $9.7 \%(13 / 134)$ & \\
\hline unknown & $4.5 \%(1 / 22)$ & & \\
\hline
\end{tabular}

2005 criteria for the differential diagnosis between typical bacterial pneumonia and atypical pneumonia.

In this study, bacteria unidentified cases was determined to be $58.1 \%(1,089 / 1,875$ patients). This result was similar to data of Saito et al (11), Ishida et al (26), Shindo et al (27). It was reflected in the detection rate of CAP in Japan.

The recent trend of increasing prevalence of macrolideresistant pneumococci in Japan prompted the JRS 2000 to develop JRS 2005 (7, 10-12, 28, 29). In the present study, the antimicrobial treatments selected were generally appropriate for diagnoses of typical bacterial pneumonia and atypical pneumonia, and clinical efficacy of greater than $80 \%$ was achieved (data not shown). Therefore, these results suggested that selection of antimicrobial treatments based on the differential diagnosis of the type of pneumonia might promote more proper use of antimicrobials $(7,8)$ and that any strategy to increase the accuracy of the differential diagnosis would be of benefit. Additional investigation is needed to clarify the optimal microbial regimen for patients with combined infection (i.e., both typical and atypical causative organisms).

In conclusion, the present study demonstrated that the JRS 2005 A-DROP system was accurate and clinically useful for the assessment of the severity of pneumonia and for the differentiation between typical and atypical pneumonia. Further, the JRS 2005 A-DROP system was simpler and clinically more practical than the PSI system, while maintaining comparable accuracy. This system may help promote proper and successful use of antimicrobials in the Japanese population.

The authors state that they have no Conflict of Interest (COI).

\section{Acknowledgement}

The authors thank Taisho Pharmaceutical Co., Ltd., Taisho
Toyama Pharmaceutical Co., Ltd., and Toyama Chemical Co., Ltd. (Tokyo, Japan) for their assistance with the statistical analyses. The authors also thank the following authorities NTT East Corporation Sapporo Hospital, Sapporo Hospital of Hokkaido Railway Company, Sapporo minami Sanjo Hospital, Sapporo Social Insurance General Hospital, Chitose City Hospital, Obihiro Kosei General Hospital, Social Welfare Corporation Hokkaido Social Work Association OBIHIRO Hospital, Hokkaido Chuo Rosai Hospital, National Hospital Organization Asahikawa Medical Center, National Hospital Organization Hakodate National Hospital, Hakodate Municipal Hospital, Hirosaki Chuo Hospital, Akita City Hospital, Yamamoto Kumiai Hospital, Morioka Red Cross Hospital, Tohoku University Graduate School of Medicine, Tohoku Kosei Nenkin Hospital, Sendai Open Hospital Sendai City Medical Center, Japanese Red Cross Sendai Hospital, South Miyagi Medical Center, Tohoku Rosai Hospital, Katta General Hospital, Yonezawa City Hospital, Sanyudo Hospital, Jusendo General Hospital, Kashima Hospital, Kureha General Hospital, Higashijyujyo Hospital, Juntendo University Nerima Hospital, Koto Hospital, The Fraternity Memorial Hospital, Kyoundo Hospital Sasaki Research Institute, Juntendo University, Eiju General Hospital, Mitsui Memorial Hospital, Toranomon Hospital, Tokyo Kouseinenkin Hospital, Kanto Central Hospital of the Mutual Aid Association of Public School Teachers, Toho University Ohashi Medical Center, Kugayama Hospital, Tokyo Kyosai Hospital, National Hospital Organization Tokyo Medical Center, Tokyo Rosai Hospital, Kyorin University School of Medicine, Machida Municipal Hospital, Showa General Hospital, Municipal Akiru Medical Center, Kawaguchi Municipal Medical Center, Saitama Red Cross Hospital, Soka Municipal Hospital, Dokkyo Medical University Koshigaya Hospital, Hanyu General Hospital, Fukaya Red Cross Hospital, Saitama Medical University Hospital, Nippon Medical School INBA-HITEC Medical Center, Saisei Hospital, Sannoh Hospital Medical Center, Juntendo University Urayasu Hospital, Tokyo Dental College Ichikawa General Hospital, Showa University Fujigaoka Hospital, Showa University Yokohama Northern Hospital, Seirei Yokohama General Hospital, Yokohama Sakae Kyosai Hospital, Yokohama City University 
Medical Center, Kawasaki General Hospital, Japan Labour Health and Welfare Organization Kanto Rosai Hospital, Fujisawa City Hospital, Kitasato University School of Medicine, Sakuramichi Clinic, Tokai University Oiso Hospital, Maebashi Red Cross Hospital, Fujioka General Hospital, Tomioka General Hospital, Gunma University Graduate School of Medicine, Tone Chuo Hospital, Keiaido Hospital, National Hospital Organization Ibarakihigashi National Hospital, National Hospital Organization Mito Medical Center, Kofu City Hospital, Ashikaga Red Cross Hospital, Utsunomiya Social Insurance Hospital, Saiseikai Utsunomiya Hospital, Iida Municipal Hospital, INA Central Hospital, Matsumoto Kyoritsu Hospital, Nagano Red Cross Hospital, Nagano Municipal Hospital, Shinrakuen Hospital, Sado General Hospital, Niigata Rinko Hospital, Tachikawa General Hospital, Nagaoka Chuo General Hospital, Niigata Prefectural Muikamachi Hospital, Niigata Rousai Hospital, Niigataken Saiseikai Sanjyo Hospital, Niigata Prefectural Kamo Hospital, Nagaoka Red Cross Hospital, Nagoya University Graduate School of Medicine, Nagoya East Municipal Medical Center, National Hospital Organization Nagoya Medical Center, Tosei General Hospital, Anjo Kosei Hospital, Toyota Kosei Hospital Aichi Prefectural Welfare Federation of Agricultural Cooperatives, Aichi Prefectual Koseiren Showa Hospital, Japanese Red Cross Nagoya Daiichi Hospital, Daiyukai General Hospital, Nagoya Ekisaikai Hospital, Mie Prefectural General Medical Center, KOMONO Hospital, Mie University Graduate School of Medicine, National Hospital Organization, Mie Chuo Medical Center, Hashima City Hospital, Gifu Prefectural General Medical Center, Nishimino Welfare Hospital, Ogaki Municipal Hospital, Yaizu City Hospital, Numazu City Hospital, Iwata City Hospital, Kanazawa Red Cross Hospital, Ishikawa-ken Saiseikai Kanazawa Hospital, Kanazawa University Hospital, Kanazawa Social Insurance Hospital, Kanazawa Medical University Hospital, Ishikawa Prefectural Central Hospital, Kaga City Hospital, Komatsu Municipal Hospital, National Hospital Organization Ishikawa Hospital, Fukui Red Cross Hospital, University of Fukui Hospital, Fukui Social Insurance Hospital, Fukuiken Saiseikai Hospital, Shinseikai Toyama Hospital, Osaka Red Cross Hospital, Nakahama Clinic, Osaka Koseinenkin Hospital, Osaka Kaisei Hospital, Aizenbashi Hospital, Higashiosaka City General Hospital, Kinki University Faculty of Medicine, PL General Hospital, Kishiwada City Hospital, Seichokai Fuchu Hospital, Tenri Hospital, Nara Prefectural Nara Hospital, Nara Prefectural Gojo Hospital, Yoshino Municipal Hospital, Nara Medical University, Naga Municipal Hospital, National Hospital Organization Minami Wakayama Medical Center, Graduate School of Medicine, Kyoto University, Kyoto First Red Cross Hospital, Shiga University of Medical Science, Shiga Medical Center for Adults, Otsu Red Cross Hospital, Kobe City Medical Center West Hospital, Kinki Central Hospital of Mutual Aid Association of Public School Teachers, National Hospital Organization Kobe Medical Center, Hyogo Prefectural Kakogawa Medical Center, National Hospital Organization Himeji Medical Center, Hiroshima Prefectural Hospital, Hiroshima Red Cross Hospital and Atomic Bomb Survirvors Hospital, JA Fuchu General Hospital, Kawasaki Medical School Kawasaki Hospital, Kurashiki Daiichi Hospital, Kaneda Hospital, Kurashiki Central Hospital, Simonoseki City Hospital, Tottori Prefectural Central Hospital, Tottori Seikyo Hospital, San-in Rosai Hospital, Kagawa Rosai Hospital, Takamatsu Red Cross Hospital, Tokushima Pre- fectural Central Hospital, Tokushima Municipal Hospital, Ehime Prefectural Central Hospital, Ehime University Graduate School of Medicine, Matsuyama Shimin Hospital, Chikamori Hospital, National Hospital Organization Kochi National Hospital, Harasanshin Hospital, Munakata Suikokai General Hospital, Japan Seamen's Relief Association Moji Hospital, Independent Administrative Agency Japan Labour Health and Welfare Organization, Kyushu Rosai Hospital, Yame General Hospital, National Hospital Organization Ureshino Medical Center, Sasebo City General Hospital, Hokusho Central Hospital, Nagasaki Rosai Hospital, Nagasaki Medical Center of Neurology, Isahaya Health Insurance General Hospital, Omura Municipal Hospital, Nagasaki University Hospital, Oita Prefecture Saiseikai Hita Hospital Social Welfare Organization, Saiseikai Imperial Gift Foundation Inc., Oita University Hospital, Oita Prefectural Hospital, Medical Corporation KEIAI-KAI Oita Nakamura Hospital, Oita Kouseiren Tsurumi Hospital, Faculty of Medical and Pharmaceutical Sciences Kumamoto University, Japan Labour Health and Welfare Organization Kumamoto Rosai Hospital, University of Miyazaki Hospital, Kagoshima Seikyo Hospital.

\section{References}

1. Lim WS, van der Eerden MM, Laing R, et al. Defining community acquired pneumonia severity on presentation to hospital: an international derivation and validation study. Thorax 58: 377-382, 2003.

2. Mandell LA, Wunderink RG, Anzueto A, et al; Infectious Diseases Society of America; American Thoracic Society. Infectious Diseases Society of America/American Thoracic Society consensus guidelines on the management of community-acquired pneumonia in adults. Clin Infect Dis 44: S27-S72, 2007.

3. Woodhead M. Community-acquired pneumonia guidelines-an international comparison: a view from Europe. Chest 113: 183S-187 S, 1998.

4. Fine MJ, Auble TE, Yealy DM, et al. A prediction rule to identify low-risk patients with community-acquired pneumonia. N Engl J Med 336: 243-250, 1997.

5. Bartlett JG, Breiman RF, Mandell LA, File TM Jr. Communityacquired pneumonia in adults: guidelines for management. The Infectious Diseases Society of America. Clin Infect Dis 26: 811-838, 1998.

6. Community-acquired Pneumonia Medical Care Guideline-Drafting Committee of the Japanese Respiratory Society. Guidelines on Respiratory Infections-Basic Concepts in the Medical Care of Community-acquired Pneumonia in Adults. 2000 (in Japanese).

7. The Committee for the Japanese Respiratory Society Guidelines in the Management of Respiratory Infections. The Japanese Respiratory Society guidelines for the management of communityacquired pneumonia in adults. Respirology 11: S79-S133, 2006.

8. The Committee for the Japanese Respiratory Society Guidelines for the Management of Respiratory Infections. The Japanese Respiratoty Society guidelines for the management of hospitalacquired pneumonia in adults 2008. Respirology 14: S1-S71, 2009.

9. Schuetz P, Koller M, Christ-Crain M, et al. Predicting mortality with pneumonia severity scores: importance of model recalibration to local settings. Epidemiol Infect 136: 1628-1637, 2008.

10. Ishida T, Hashimoto T, Arita M, Tojo Y, Tachibana H, Jinnai M. A 3-year prospective study of a urinary antigen-detection test for Streptococcus pneumoniae in community-acquired pneumonia: utility and clinical impact on the reported etiology. J Infect Chemother 10: 359-363, 2004.

11. Saito A, Kohno S, Matsushima $\mathrm{T}$, et al. Prospective multicenter 
study of the causative organisms of community-acquired pneumonia in adults in Japan. J Infect Chemother 12: 63-69, 2006.

12. Goto H, Shimada K, Ikemoto H, Oguri T; Study Group on Antimicrobial Susceptibility of Pathogens Isolated from Respiratory Infections. Antimicrobial susceptibility of pathogens isolated from more than 10,000 patients with infectious respiratory diseases: a 25-year longitudinal study. J Infect Chemother 15: 347-360, 2009.

13. Usui K, Tanaka Y, Noda H, Ishihara T. Comparison of three prediction rules for prognosis in community acquired pneumonia: Pneumonia Severity Index (PSI), CURB-65, and A-DROP. Nihon Kokyuki Gakkai Zasshi 47: 781-785, 2009 (in Japanese).

14. Tashiro M, Fukushima K, Hara A, et al. Evaluation of the severity of community-acquired pneumonia based on the JRS and IDSA/ ATS guidelines. Nihon Kokyuki Gakkai Zasshi 46: 981-986, 2008 (in Japanese).

15. Niederman MS. Making sense of scoring systems in community acquired pneumonia. Respirology 14: 327-335, 2009.

16. Gomi K, Miki M, Itabashi S, et al. Survey of the actual condition of community-acquired pneumonia and the results of validation of the new and old guidelines on medical care of communityacquired pneumonia. Nihon Kokyuki Gakkai Zasshi 45: 836-843, 2007 (in Japanese).

17. Niederman MS, Feldman C, Richards GA. Combining information from prognostic scoring tools for CAP: an American view on how to get the best of all worlds. Eur Respir J 27: 9-11, 2006.

18. Aujesky D, Auble TE, Yealy DM, et al. Prospective comparison of three validated prediction rules for prognosis in communityacquired pneumonia. Am J Med 118: 384-392, 2005.

19. Charles PG, Wolfe R, Whitby M, et al. SMART-COP: a tool for predicting the need for intensive respiratory or vasopressor support in community-acquired pneumonia. Clin Infect Dis 47: 375-384, 2008.

20. España PP, Capelastegui A, Gorordo I, et al. Development and validation of a clinical prediction rule for severe community- acquired pneumonia. Am J Respir Crit Care Med 174: 1249-1256, 2006.

21. Myint PK, Kamath AV, Vowler SL, Maisey DN, Harrison BD; British Thoracic Society. Severity assessment criteria recommended by the British Thoracic Society (BTS) for communityacquired pneumonia (CAP) and older patients. Should SOAR (systolic blood pressure, oxygenation, age and respiratory rate) criteria be used in older people? A compilation study of two prospective cohorts. Age Ageing 35: 286-291, 2006.

22. Seki M, Watanabe A, Mikasa K, Kadota J, Kohno S. Revision of the severity rating and classification of hospital-acquired pneumonia in the Japanese Respiratory Society guidelines. Respirology 13: $880-885,2008$.

23. Kollef MH, Morrow LE, Baughman RP, et al. Health careassociated pneumonia (HCAP): a critical appraisal to improve identification, management, and outcomes-proceedings of the HCAP Summit. Clin Infect Dis 46: S296-S334, 2008.

24. Sakamoto K. An international comparative analysis of Japanese medical insurance policies. Kawasaki Medical Welfare Journal 15: 471-484, 2006 (in Japanese).

25. Abe T. Nihon no Iryou Seido. NLI Research Institute, 2008: 2835 (in Japanese).

26. Ishida $T$, Hashimoto $T$, Arita M, Ito I, Osawa M. Etiology of Community-acquired pneumonia in hospitalized patients: A 3-Year Prospective Study in Japan. Chest 114: 1588-1593, 1998.

27. Shindo Y, Sato S, Maruyama E, et al. Health-care-associated pneumonia among hospitalized patients in a Japanese community hospital. Chest 135: 633-640, 2009.

28. Ikemoto H, Watanabe $\mathrm{K}$, Mori $\mathrm{T}$, et al. Susceptibilities of bacteria isolated from patients with lower respiratory infectious diseases to antibiotics (1996). Jpn J Antibiot 51: 437-474, 1998 (in Japanese).

29. Ikemoto H, Arakawa M, Gejyo F, et al. Susceptibilities of bacteria isolated from patients with lower respiratory infectious diseases to antibiotics (1998). Jpn J Antibiot 53: 261-298, 2000 (in Japanese).

(C) 2011 The Japanese Society of Internal Medicine http://www.naika.or.jp/imindex.html 